\title{
Nomogram: An analogue tool to deliver digital knowledge
}

\author{
Seo Young Park, PhD
}

\footnotetext{
From the Division of General Internal Medicine, Department of Medicine, University of Pittsburgh School of Medicine, Pittsburgh, Pa.

Disclosures: Author has nothing to disclose with regard to commercial support.

Received for publication Dec 13, 2017; accepted for publication Dec 21, 2017; available ahead of print Jan 19, 2018.

Address for reprints: Seo Young Park, PhD, 200 Meyran Ave, Suites 300, Pittsburgh, PA 15213 (E-mail: syp8@ pitt.edu).

J Thorac Cardiovasc Surg 2018;155:1793

$0022-5223 / \$ 36.00$

Copyright (C) 2018 by The American Association for Thoracic Surgery

https://doi.org/10.1016/j.jtcvs.2017.12.107
}

In this issue of the Journal, Qixing and colleagues ${ }^{1}$ developed a prediction model for overall survival of stage IIIAN2 non-small cell lung cancer after surgery. The model was built on the basis of the Surveillance, Epidemiology, and End Results database using the Cox proportional hazard model and validated in separate data from Jiangsu Cancer Hospital Lung Cancer Center. In addition to reporting the beta coefficients and hazard ratios of the predictors in the final model, they provided a nomogram to present their model.

A nomogram is a graphical tool that is designed to approximate complicated calculation quickly and without a computer or calculator. It was invented in the 19th century and flourished before the time when calculators and computers became readily available. However, with the advent of the digital era, the nomogram lost its popularity and was not needed anymore. Now we can perform complicated computation more quickly, and high-performance computers became smaller in size and more readily available. Even smartphone applications can provide faster and more accurate calculation than a nomogram can.

So, did the advance in technology improve health care? Surely it did in many ways, especially in terms of clinical prediction modeling. Now we can use more sophisticated methodologies and bigger data than we used to be able to handle, resulting in more accurate, precise, and generalizable prediction models. However, the complexity of the model often makes it difficult to interpret and hinders clinicians from using the model in practice. To combat this problem and make the models more usable by clinicians, sometimes the models are modified into a simpler scoring system, or the Classification and Regression Trees method is used. These are valid and useful methods with their own drawbacks: One may introduce some bias by simplifying the prediction model to make an easy-to-calculate

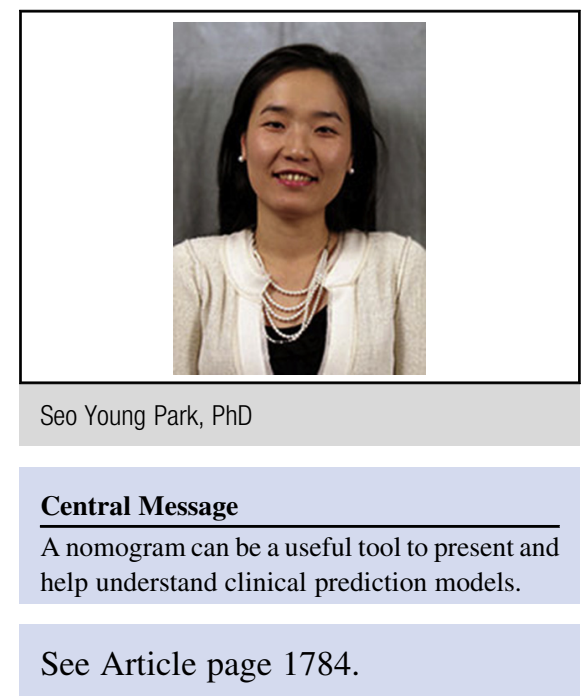

scoring system, and Classification and Regression Trees usually require big data to generate reliable and generalizable model and tend to be less accurate than other approaches.

This is the point where a nomogram can play an important role again in this digital age. By graphically representing the effect of each predictor on the outcome, it gives readers a more tangible interpretation of each predictor's impact on the outcome. Looking at the nomogram in the article by Mao and colleagues, ${ }^{1}$ a clinician can eyeball the sum of all predictors' effect for a given patient and predict the probability of 1-, 3-, and 5-year survivals. This is easier than programming the formula in the computer beforehand or pulling out a phone and typing all the beta coefficients manually, which is likely to induce mistakes.

After all, it matters how we humans understand and use the knowledge as much as how we became better at gaining that knowledge. In that sense, something as analogue as the nomogram can still be instrumental to disseminate knowledge and improve health care. I hope to see more articles using nomograms to deliver their findings.

\section{Reference}

1. Mao Q, Xia W, Dong G, Chen S, Wang A, Jin G, et al. A nomogram to predict the survival of stage IIIA-N2 non-small cell lung cancer after surgery. J Thorac Cardiovasc Surg. 2018;155:1784-92.e3. 